\title{
Design and Application of Intelligent Tourism System under the Background of Cloud Computing Information Technology
}

\author{
Lei Jie \\ Business School, Yulin Normal University, Yulin, Guangxi, China \\ alex.lei.alex@qq.com
}

Keywords: Intelligent tourism; big data; cloud computing.

\begin{abstract}
In order to realize the function of distributing tourism service resources and provide better services for tourists, on the basis of fully understanding the definition of cloud computing and the main key technologies, the tourists' needs are firstly analyzed, and the overall design scheme of intelligent tourism system is clarified. Cloud computing information technology, Unity 3D software, and augmented reality and mobile phone VR technology are used to model. A set of intelligent tourism system with tourism service, mobile guide and consultation as the main modules is developed and designed based on Android platform. The system can run safely and steadily after being tested on the cloud platform.
\end{abstract}

\section{Research background}

"Intelligent tourism" is mainly building a smart scenic spot to manage the scenic spot in a dynamic and delicate way, achieve intelligent application, and upgrade the industry as a whole. Through the full application of new generation of IT technology to the various links of the tourism industry chain, the intelligent state of the whole process of tourism is achieved [1]. Intelligent tourism cannot develop without big data. It needs to rely on big data to provide enough favourable resources, so that the development of intelligent tourism can be "intelligent". Data accurately reflect where the city's tourist market is and which products are of concern to tourists, which provides an important data support for precision marketing and has a subversive effect on decision-making marketing [2]. Although big data is a good thing and has a broad application prospect in the tourism industry, there are still great obstacles to the application of big data in the whole industry. These obstacles come from data collection and data analysis and mining.

$\mathrm{Li}$ et al. [3] pointed out that cloud computing can help transport systems cope with the large amount of storage and computing resources needed for efficient use of traffic policy agents and mass transfer of data, and show the state of traffic control and management systems based on mobile multi-agent technology. Intelligent traffic cloud can provide decision support, standard development environment such as traffic management strategy and so on. The Internet of Things has the potential to provide SCC with ubiquitous connectivity devices and smart sensor networks, and large data analysis has the potential to achieve real-time control from the Internet of Things to SCC. Therefore, Sun et al. [4] regarded mobile crowdsourcing and network physical cloud computing as two of the most important Internet of Things technologies to promote SCC, and used TreSight to conduct the Internet of Things and large data analysis of intelligent tourism and sustainable cultural heritage in Trento, Italy. Li [5], through the use of cloud information technology, studied and proposed an automatic road generation method for complex terrain, that is, a road generation algorithm based on Voronoi map. This method can automatically generate streets with the same uniform changes as the surrounding terrain and environment under complex terrain conditions such as elevation and river, and enriches the research contents of virtual city road modeling. Zhou et al. [6] put forward an intelligent tourism cloud solution based on cloud computing. Three cloud-based models, such as IaaS (Infrastructure as a Service), PaaS (Platform as a Service) and SaaS (Software as a Service), are used to virtualize tourism resources and construct an intelligent tourism cloud platform. Specifically, IaaS is used to integrate and allocate tourism 
resources; PaaS is used for achieving reuse and virtualization; and SaaS is used for customization and leasing. Openstack and Hadoop open source software based on cloud computing are used in platform design to transform tourism resources into intelligent tourism cloud systems, which have the characteristics of unified management, sales, processing and service. Agent-based traffic management systems can use autonomy, mobility and adaptability of mobile agents to handle dynamic traffic environments.

To sum up, the research on intelligent information system based on "big data" can effectively solve the problem of comprehensive perception, rapid processing and efficient storage of real information data. Therefore, a set of intelligent tourism service system combined with big data is designed to collect, analyze and integrate big data to realize the function of allocating tourism service resources, and to provide a certain reference for tourists.

\section{Related technology introduction}

\subsection{Definition of cloud computing}

Cloud computing is considered a new enterprise IT architecture; Intel defines cloud computing from a hardware perspective; Google defines cloud computing as a new data storage and computing model, data center. CCA believes that cloud computing can be defined as aggregating computing as a tool and software, namely a service, and proposes that cloud computing is the delivery of services by applications over the Internet and the provision of these services by hardware and system software in data centers [7].

Cloud computing is a new computing model based on distributed computing, parallel computing and grid computing. It provides reliable and secure data storage, powerful computing power and convenient Internet services. Cloud computing technology has two meanings. On the one hand, it refers to the system platform used to construct applications, which is equivalent to the operating system on personal computers, called cloud computing platform; on the other hand, it describes cloud computing applications based on this platform (called cloud applications). To understand cloud computing in a more general way, it is to create a pool of resources that provide the resources needed for an application and provide features such as schedulability, high availability, and failover.

\subsection{Cloud computing type}

Cloud computing is mainly divided into three layers, namely application layer, platform layer and infrastructure layer. Different layers provide different services and these services constitute the corresponding subset of cloud services. The application layer is at the top of the architecture and consists of the actual cloud applications [8]. The platform layer is made up of operation system, application framework and so on. The infrastructure layer is responsible for managing the hardware resources in the cloud. The service level of cloud computing is shown in Figure 1.

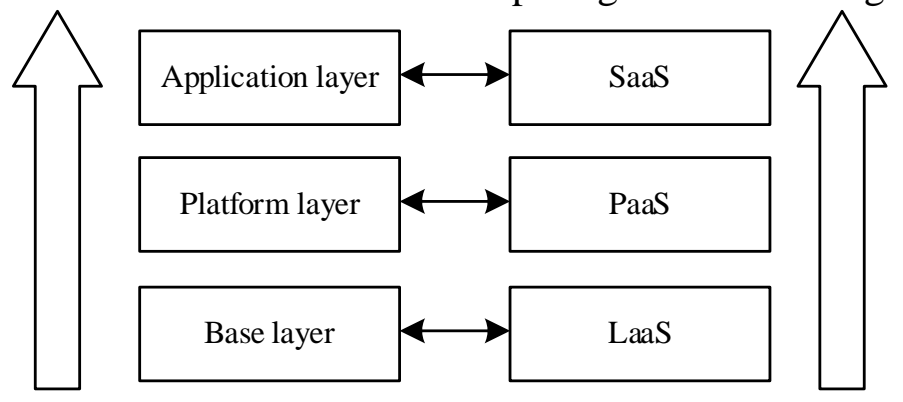

Figure 1 Service level of cloud computing

Cloud computing can be divided into IaaS, PaaS and SaaS. Cloud computing service model includes "end", "management" and "cloud" three levels, "end" refers to any terminal equipment that can access the "cloud" and complete the information exchange; "management" refers to the communication network used to complete the user information transmission; "cloud" is the way to achieve business, that is, business mode. 


\subsection{Key technologies of cloud computing}

\subsubsection{Hadoop ecosystem}

Catalysts for the shift in thinking patterns are the birth of a host of new technologies that can address the three challenges posed by big data analysis. Hadoop, rooted in the open source community, is the most widely used technology on big data platforms today, especially for unstructured data such as text, social media subscriptions, and video. In addition to distributed file systems, along with Hadoop comes the MapReduce architecture for processing big data sets. According to authoritative reports, many companies are using or evaluating Hadoop technology as a standard for their big data platforms [9].

\subsubsection{NoSQL database}

On the basis of a generalized faction, the NoSQL database itself contains several technologies. Overall, they focus on the constraints of relational database engines, such as indexing, streaming media, and high-traffic Web services. In these areas, the efficiency of NoSQL is significantly higher than that of relational database engine [10].

\section{System design and Implementation}

\subsection{Requirement analysis}

Tourist use case diagram is shown in Figure 2. The demand for tourists is mainly as follows: first, tourists can obtain tourist information through a variety of terminals such as PC, mobile phone and tablet. At the same time, they can scan the two-dimensional code of Weixin and pay attention to the public number of the scenic spot. They can not only browse the basic information of the scenic spot, but also get the latest information in time; second, online booking accommodation visitors can purchase the scenic spot tickets online, but also can book a hotel, convenient for tourists to travel; third, electronic guided tourists can download the scenic area APP. The electronic map is not only convenient for tourists to visit, but also help them timely upload tourist information, which provides security for tourists. When tourists visit scenic spots, they can also listen to the explanation, so that tourists better understand the history and culture of the scenic area.

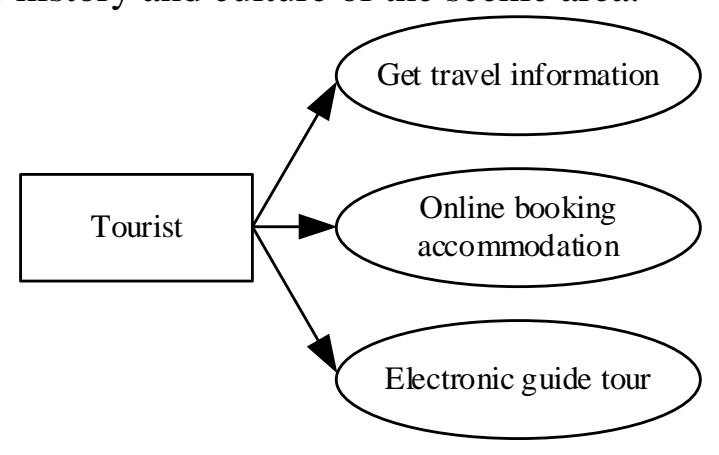

Figure 2 Tourist use case diagram

\subsection{System overall design}

Through the analysis of the current situation of China's tourism industry, the system is in the form of modules (i.e. intelligent management system module, panoramic virtual reality system module, passenger flow forecasting system module and electronic navigation system module etc.), as shown in Figure 3. The functions of each module are effectively combined, and the data standards and interfaces of each module are unified through the management system to achieve effective data collection and analysis to achieve construction goals. 


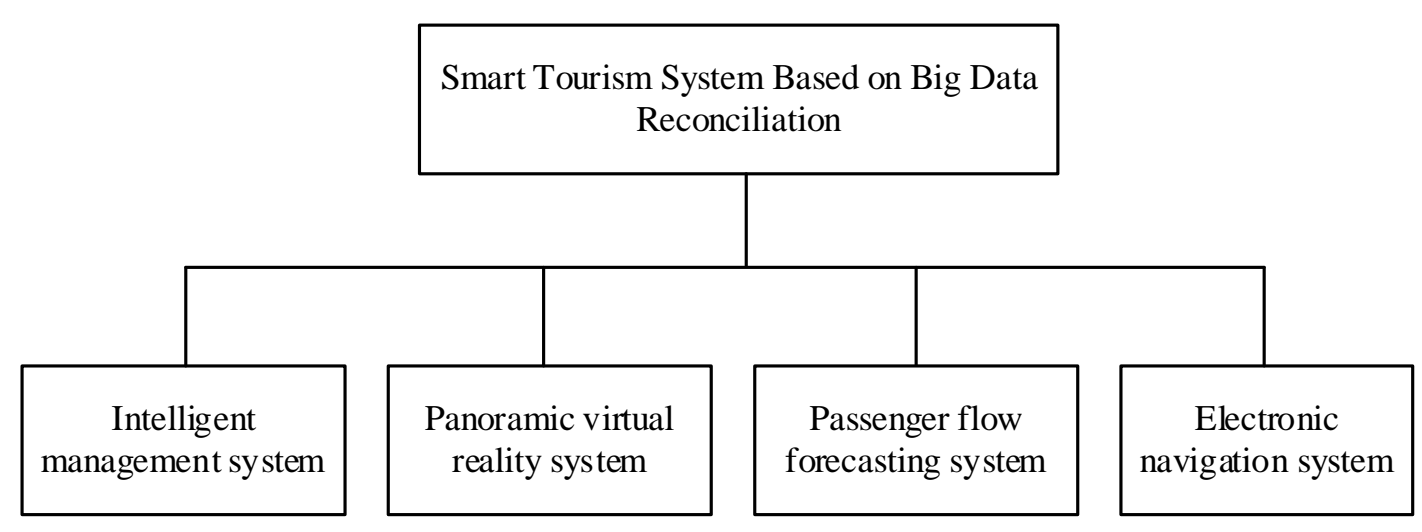

Figure 3 Design and implementation plan of intelligent tourism information system

The first module: Intelligent management system includes front-end display pages and background management system. The front-end display pages include information release, scenic spot display, booking tickets, hotels and other functions, aimed at intelligently and conveniently servicing the tourists. The background management system includes information editing, order management and passenger flow statistics report forms.

The second module: panoramic virtual reality system mainly focuses on scenic spot display and virtual experience. It uses the popular panoramic photography technology and 3D modeling technology to achieve the effect of street map for scenic spots, so that users can experience the scene and know more about the scenic spots they are interested in.

The third module: passenger flow forecasting system mainly includes passenger flow data collection, statistics, analysis and prediction. Using big data technology, combined with historical data and real-time weather and holiday information, passenger flow data is predicted, which provides a reference for tourists to travel. Through the passenger flow statistics and early warning system, the early warning of passenger flow can be achieved. It can provide accurate passenger flow information for site operators, timely detect major potential safety hazards of passenger flow, help the field managers in peak passenger flow period to take appropriate measures to guide passenger flow correctly and reasonably, balance the pressure of scenic area passenger flow, and avoid accidents.

The fourth module: electronic navigation system is through the combination of mobile Internet, Internet of Things and multimedia technology, to develop Android and IOS version of APP and creat a public number based on Weixin. Users use the client or Weixin electronic map real-time positioning automatically through voice navigation to explain; at the same time, it simultaneously displays illustration point pictures and video information, which liberates tourists who must follow the tour guide in order to understand the information of scenic spots, achieves personalized tours, recommends to tourists concerned about scenic spots, and enhances the tourist experience.

\subsection{Key technologies of the system}

The system is developed based on Android platform, compatible with Android 2.2 or more systems. 3D model uses Unity 3D software for modeling, as well as augmented reality and mobile phone VR technology. Maps are developed based on Baidu Map SDK to provide LBS services for tourists. The system can customize the three-dimensional landscape and guide information of different scenic spots for the subject customers. Besides the pictures, words and videos introduction to the scenic spots, the system also adds new forms such as voice explanation, three-dimensional guide and interesting tasks to enable users to understand the information of the scenic spots in an all-round way and integrate into the activities of the scenic spots. Through history story, near scenic spots, photo sharing and other contents, the cultural connotations are enriched and the navigation system experience chain is deepened. The exquisite 3D model and animation, precise positioning and interactive experience, novel intelligent mode and portal function can fully meet the actual needs of tourists in the scenic area to understand human information, social interaction, positioning navigation and route setting, and increase the cultural and interesting nature of tourism. 


\subsection{System design}

The implementation framework of this system is based on MVC mode, which can be subdivided into view layer, data layer and control layer, whose structure is shown in Figure 4. The main function of the view layer is to display the content of the interface on the mobile client and receive the user's action on the interface. The main function of the control layer is to execute the user's operation and control the related business logic processing. It can not only extract the local cached data from the data layer, but also access the cloud data services through the mobile Internet interface. The main function of the data layer is to maintain the cache of local files, which mainly stores basic data of scenic spots, image caching and other small files.

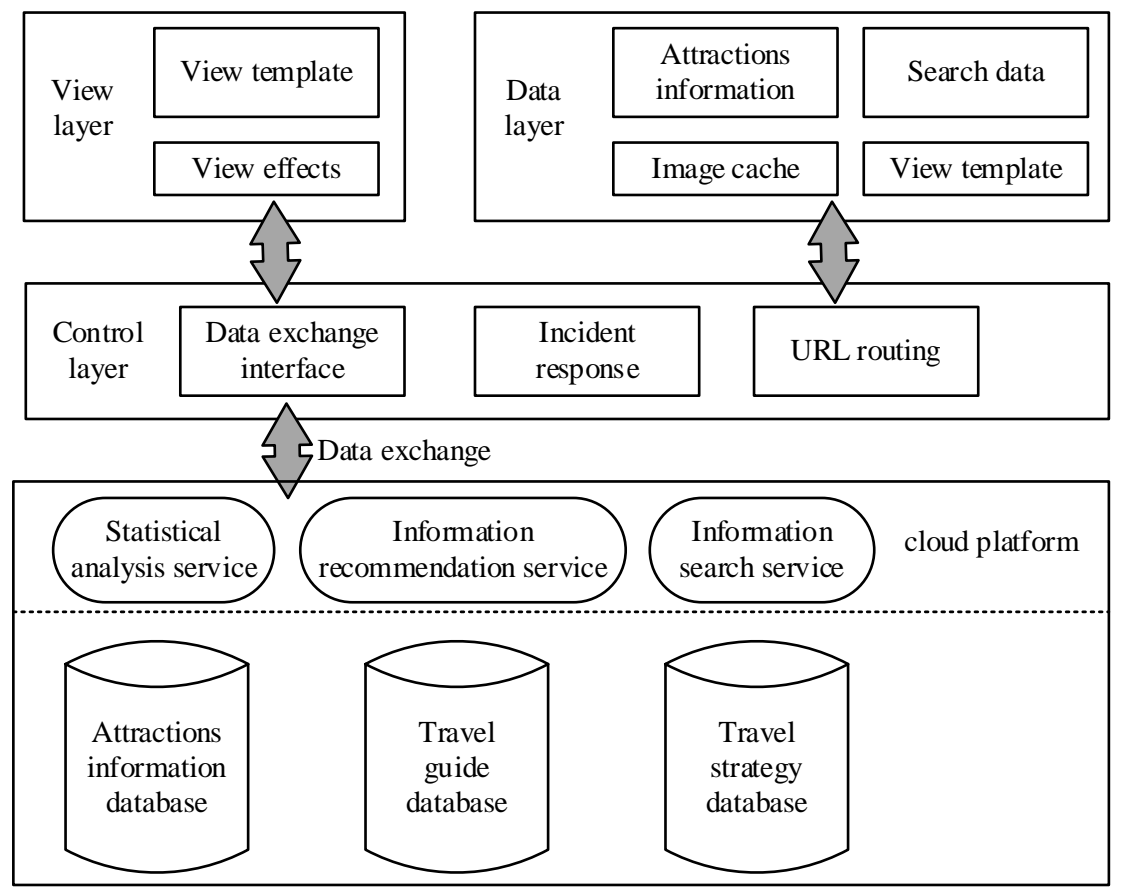

Figure 4 Intelligent tourism system architecture

With the popularity of mobile Internet, more and more users begin to choose to use intelligent terminals. In order to facilitate the majority of tourists to enjoy better service, users only need to scan two-dimensional code in the site or scenic spots, and can pay attention to Changbai Mountain Intelligent Tourism Public Number in Weixin, which includes tourism services, mobile navigation, and consultation three parts. The user interface relationship is shown in Figure 5.

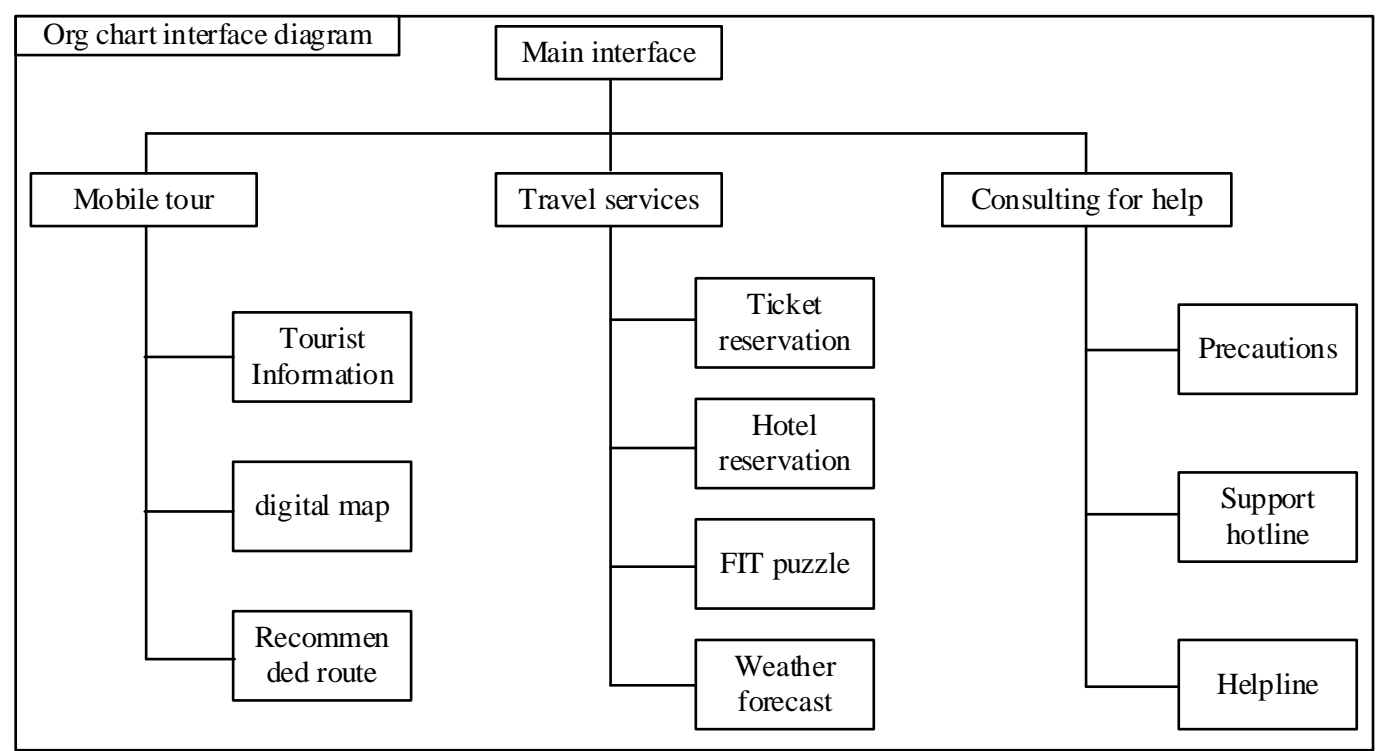

Figure 5 Interface relationship diagram of intelligent tourism system 


\subsubsection{Tourism service}

The tour services include ticket booking, hotel reservation, individual matching, and weather forecast. Tourists can choose ticket booking, hotel reservation, individual matching, etc, they can also choose the appropriate ticket, hotel, and tour group, and the use of Wechat payment order information will take effect. Weather forecasts are provided for tourists especially so that visitors can check the real time weather. This function module is mainly based on ticket booking, and the function flow chart of ticket booking is shown in Figure 6.

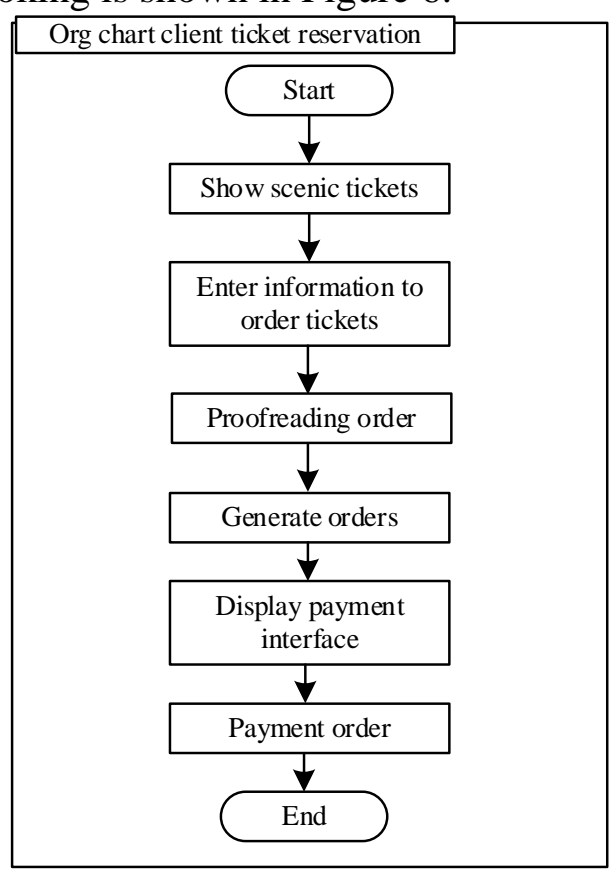

Figure 6 Client ticket booking route map of intelligent tourism system

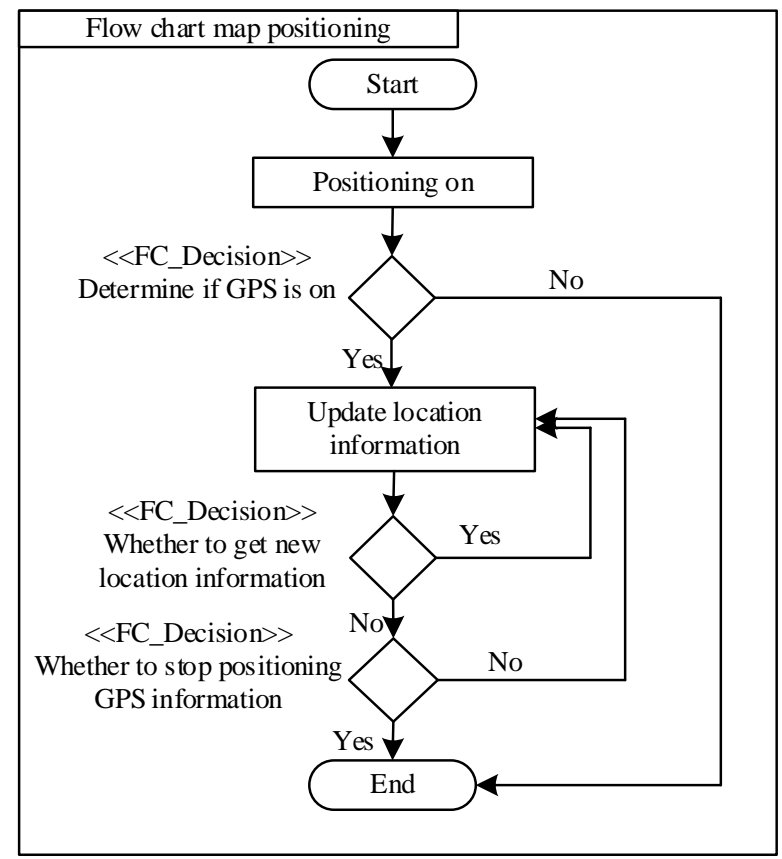

Figure 7 Mobile map flow chart of intelligent tourist system

\subsubsection{Mobile navigation}

Mobile navigation includes the introduction to scenic spots, electronic map and recommended route and other functions. The introduction to scenic spots includes text and video explanation of all the scenic spots of Changbai Mountain. When tourists visit the corresponding scenic spots, they can choose the corresponding scenic spots introduction. It can let tourists know the history of each 
scenic spot in detail and solve the problem that tourists must rely on the guide. Visitors click on the electronic map to view the map of the scenic area, and check the real-time location through positioning. The system can recommend routes for tourists according to real-time passenger flow information and tourist interest. Visitors can click on the electronic map to locate the current location, providing navigation services for visitors. The flow chart is shown in Figure 7.

\subsubsection{Advisory help}

Advisory help includes tourism attention, consultation hotline and help line. Attentions are mainly to remind tourists of tourism attention in Changbai Mountain, reduce the occurrence of accidents, and provide help for the safety of tourists. Visitors can call the hotline no matter what problems they encounter in the scenic spot. Customer service will provide specific help to the visitors. This module only needs to be set as a static interface.

\subsection{System test}

Through Ali cloud computing technology and service platform, the system is tested. The system purchased Aliyun cloud server ECS, relational database service RDS, open storage service OSS, content distribution network CDN and other product services for test running, and the results show that the system can run safely and steadily.

\section{Conclusion}

Internet is a new tool for public entrepreneurship and innovation. The representative of the "Internet +" era of tourism is intelligent tourism. Nowadays, more and more people choose to go out of their homes and tourism is becoming a new way of life. Intelligent tourism system based on big data can not only provide services for tourists, but also facilitate management. It is also the inevitable requirement of the development of social informatization. This research combines the latest "cloud computing" theory and technology to design intelligent tourism system, and completes the mobile intelligent navigation system. Finally, through the system test, it is found that the system can operate safely and stably in the Ali cloud platform.

\section{Acknowledgement}

The project was supported by the Research Project Of Yulin Normal University In 2018. Research On Pattern Construction Of Smarter Tourism On Online And Offline Collaboration Integration In The "Internet +" Era (2018YJKY09).

\section{References}

[1] Liu H, Li J, Peng J. A novel recommendation system for the personalized smart tourism route: Design and implementation [J], International Conference on Cognitive Informatics and Cognitive Computing. IEEE, 2015:291-296.

[2] Byun J, Kim B W, Chang Y K, et al. 4G LTE network access system and pricing model for IoT MVNOs: spreading smart tourism [J]. Multimedia Tools \& Applications, 2016, 76(19):1-24.

[3] Li Z J, Chen C, Wang K. Cloud Computing for Agent-Based Urban Transportation Systems [J]. IEEE Intelligent Systems, 2011, 26(1):73-79.

[4] Sun Y, Song H, Jara A J, et al. Internet of Things and Big Data Analytics for Smart and Connected Communities [J]. IEEE Access, 2016, 4:766-773.

[5] Li M. Research on the mechanism and influence factors of urban style building based on cloud computing logistics information [J]. Cluster Computing, 2018(2):1-8.

[6] Zhou X, Ma. A solution to wisdom tourism cloud based on cloud computing [J]. Journal of Southeast University, 2012, 42:261-264. 
[7] Aminsoofi A, Irfan Khan M, Fazaleamin F A. A Review on Data Security in Cloud Computing [J]. International Journal of Computer Applications, 2017, 96(2):95-96.

[8] Zhang Y Q, Wang X F, Liu X F, et al. Survey on Cloud Computing Security[J]. Journal of Software, 2016, 8271(1):302 - 311.

[9] Lin H K, Harding J A, Chen C I. A Hyperconnected Manufacturing Collaboration System Using the Semantic Web and Hadoop Ecosystem System [J]. Procedia Cirp, 2016, 52:18-23.

[10] Lh S S D A, Costa O M. A Medical Image Backup Architecture Based on a NoSQL Database and Cloud Computing Services [J]. Stud Health Technol Inform, 2014, 216:929. 